Article

\title{
Neutrosophic Quadruple BCI-Positive Implicative Ideals
}

\author{
Young Bae Jun ${ }^{1}$, Seok-Zun Song ${ }^{2, *}$ and Seon Jeong Kim ${ }^{3}$ \\ 1 Department of Mathematics Education, Gyeongsang National University, Jinju 52828, Korea; \\ skywine@gmail.com \\ 2 Department of Mathematics, Jeju National University, Jeju 63243, Korea \\ 3 Department of Mathematics, Natural Science of College, Gyeongsang National University, Jinju 52828, \\ Korea; skim@gnu.ac.kr \\ * Correspondence: szsong@jejunu.ac.kr
}

Received: 4 April 2019; Accepted: 25 April 2019; Published: 28 April 2019

check for updates

\begin{abstract}
By considering an entry (i.e., a number, an idea, an object, etc.) which is represented by a known part $(a)$ and an unknown part $(b T, c I, d F)$ where $T, I, F$ have their usual neutrosophic logic meanings and $a, b, c, d$ are real or complex numbers, Smarandache introduced the concept of neutrosophic quadruple numbers. Using the concept of neutrosophic quadruple numbers based on a set, Jun et al. constructed neutrosophic quadruple BCK/BCI-algebras and implicative neutrosophic quadruple BCK-algebras. The notion of a neutrosophic quadruple BCI-positive implicative ideal is introduced, and several properties are dealt with in this article. We establish the relationship between neutrosophic quadruple ideal and neutrosophic quadruple BCI-positive implicative ideal. Given nonempty subsets $I$ and $J$ of a BCI-algebra, conditions for the neutrosophic quadruple $(I, J)$-set to be a neutrosophic quadruple BCI-positive implicative ideal are provided.
\end{abstract}

Keywords: neutrosophic quadruple BCK/BCI-number; neutrosophic quadruple BCK/BCI-algebra; neutrosophic quadruple (BCI-positive implicative) ideal

MSC: 06F35; 03G25; 08A72

\section{Introduction}

A BCK/BCI-algebra is a class of logical algebras introduced by K. Iséki (see $[1,2]$ ) and was extensively investigated by several researchers. Neutrosophic algebraic structures in $\mathrm{BCK} / \mathrm{BCI}$-algebras are discussed in the papers [3-10]. Smarandache introduced the notion of neutrosophic sets with wide applications in sciences (see [11-13]), which is a more general stage to extend the notions of classic set, (intuitionistic) fuzzy set and interval valued (intuitionistic) fuzzy set. Smarandache [14] introduced the concept of neutrosophic quadruple numbers by considering an entry (i.e., a number, an idea, an object, etc.) which is represented by a known part $(a)$ and an unknown part $(b T, c I, d F)$, where $T, I, F$ have their usual neutrosophic logic meanings and $a, b, c, d$ are real or complex numbers. Using the notion of neutrosophic quadruple numbers based on a set, Jun et al. [15] constructed neutrosophic quadruple BCK/BCI-algebras and implicative neutrosophic quadruple BCK-algebras (see also [16]).

In this paper, we introduce the notion of a neutrosophic quadruple BCI-positive implicative ideal, and investigate several properties. We consider relations between neutrosophic quadruple ideal and neutrosophic quadruple BCI-positive implicative ideal. Given nonempty subsets $I$ and $J$ of a BCI-algebra $U$, we provide conditions for the neutrosophic quadruple $(I, J)$-set to be a neutrosophic quadruple BCI-positive implicative ideal. 


\section{Preliminaries}

A BCI-algebra is a set $U$ with a special element 0 and a binary operation $*$ that satisfies the following conditions:

(I) $\quad(\forall p, q, r \in U)(((p * q) *(p * r)) *(r * q)=0)$,

(II) $(\forall p, q \in U)((p *(p * q)) * q=0)$,

(III) $(\forall p \in U)(p * p=0)$,

(IV) $(\forall p, q \in U)(p * q=0, q * p=0 \Rightarrow p=q)$.

If a BCI-algebra $U$ satisfies the following identity:

(V) $(\forall p \in U)(0 * p=0)$,

then $U$ is called a $B C K$-algebra. In a BCK/BCI-algebra $U$, the following conditions are valid.

$$
\begin{aligned}
& (\forall p \in U)(p * 0=p), \\
& (\forall p, q, r \in U)(p \leq q \Rightarrow p * r \leq q * r, r * q \leq r * p), \\
& (\forall p, q, r \in U)((p * q) * r=(p * r) * q), \\
& (\forall p, q, r \in U)((p * r) *(q * r) \leq p * q)
\end{aligned}
$$

where $p \leq q$ if and only if $p * q=0$.

Any BCI-algebra $U$ satisfies the following conditions (see [17]):

$$
\begin{aligned}
& (\forall p, q \in U)(p *(p *(p * q))=p * q) \\
& (\forall p, q \in U)(0 *(p * q)=(0 * p) *(0 * q)) \\
& (\forall p, q \in U)(0 *(0 *(p * q))=(0 * q) *(0 * p)) .
\end{aligned}
$$

By a subalgebra of a BCK/BCI-algebra $U$, we mean a nonempty subset $S$ of $U$ such that $p * q \in S$ for all $p, q \in S$. We say that a subset $G$ of a BCK/BCI-algebra $U$ is an ideal of $U$ if it satisfies:

$$
\begin{aligned}
& 0 \in G, \\
& (\forall p \in U)(\forall q \in G)(p * q \in G \Rightarrow p \in G) .
\end{aligned}
$$

A subset $G$ of a BCI-algebra $U$ is called a BCI-positive implicative ideal of $U$ (see $[18,19])$ if it satisfies (8) and

$$
(\forall p, q, r \in U)(((p * r) * r) *(q * r) \in G, q \in G \Rightarrow p * r \in G),
$$

For further information regarding BCK/BCI-algebras and neutrosophic set theory, we refer the reader to the books $[17,20]$ and the site $[21]$ respectively. We will use neutrosophic quadruple numbers based on a set instead of real or complex numbers.

Let $U$ be a set. A neutrosophic quadruple $U$-number is an ordered quadruple $(a, p T, q I, r F)$, where $a, p, q, r \in U$ and $T, I, F$ have their usual neutrosophic logic meanings (see [15]).

The set of all neutrosophic quadruple $U$-numbers which is denoted by $\mathcal{N}(U)$, that is,

$$
\mathcal{N}(U):=\{(a, p T, q I, r F) \mid a, p, q, r \in U\},
$$

is called the neutrosophic quadruple set based on $U$. In particular, if $U$ is a BCK/BCI-algebra, then a neutrosophic quadruple $U$-number is called a neutrosophic quadruple $B C K / B C I$-number and $\mathcal{N}(U)$ is called the neutrosophic quadruple BCK/BCI-set.

We define a binary operation $\square$ on the neutrosophic quadruple BCK/BCI-set $\mathcal{N}(U)$ by

$$
(a, p T, q I, r F) \boxminus(b, u T, v I, w F)=(a * b,(p * u) T,(q * v) I,(z * w) F)
$$


for all $(a, p T, q I, r F),(b, u T, v I, w F) \in \mathcal{N}(U)$. Given $a_{1}, a_{2}, a_{3}, a_{4} \in U$, the neutrosophic quadruple BCK/BCI-number $\left(a_{1}, a_{2} T, a_{3} I, a_{4} F\right)$ is denoted by $\tilde{a}$, that is,

$$
\tilde{a}=\left(a_{1}, a_{2} T, a_{3} I, a_{4} F\right),
$$

and the neutrosophic quadruple BCK/BCI-number $(0,0 T, 0 I, 0 F)$ is denoted by 0 , that is,

$$
\tilde{0}=(0,0 T, 0 I, 0 F),
$$

which is called the zero neutrosophic quadruple $B C K / B C I$-number. Then $(\mathcal{N}(U)$; $\square, \tilde{0})$ is a $\mathrm{BCK} / \mathrm{BCI}$-algebra (see [15]), which is called neutrosophic quadruple BCK/BCI-algebra, and it is simply denoted by $\mathcal{N}(U)$.

We define an order relation " $\ll$ " and the equality " $=$ " on the neutrosophic quadruple BCK/BCI-algebra $\mathcal{N}(U)$ as follows:

$$
\begin{aligned}
& \tilde{p} \ll \tilde{q} \Leftrightarrow p_{i} \leq q_{i} \text { for } i=1,2,3,4, \\
& \tilde{p}=\tilde{q} \Leftrightarrow p_{i}=q_{i} \text { for } i=1,2,3,4
\end{aligned}
$$

for all $\tilde{p}=\left(p_{1}, p_{2} T, p_{3} I, p_{4} F\right), \tilde{q}=\left(q_{1}, q_{2} T, q_{3} I, q_{4} F\right) \in \mathcal{N}(U)$. It is easy to verify that " $\ll$ " is an equivalence relation on $\mathcal{N}(U)$.

Let $U$ be a BCK/BCI-algebra. Given nonempty subsets $I$ and $J$ of $U$, consider the set

$$
\mathcal{N}(I, J):=\{(a, p T, q I, r F) \in \mathcal{N}(U) \mid a, p \in I \& q, r \in J\},
$$

which is called the neutrosophic quadruple $(I, J)$-set.

The neutrosophic quadruple $(I, J)$-set $\mathcal{N}(I, J)$ with $I=J$ is denoted by $\mathcal{N}(I)$, and it is called the neutrosophic quadruple I-set.

\section{Neutrosophic Quadruple BCI-Positive Implicative Ideals}

In what follows, let $U$ and $\mathcal{N}(U)$ be a BCI-algebra and a neutrosophic quadruple BCI-algebra, respectively, unless otherwise specified.

Definition 1. Given nonempty subsets I and J of $U$, if $\mathcal{N}(I, J)$ is a BCI-positive implicative ideal of $\mathcal{N}(U)$, we say $\mathcal{N}(I, J)$ is a neutrosophic quadruple BCI-positive implicative ideal of $\mathcal{N}(U)$.

Example 1. Consider a BCI-algebra $U=\{0,1, a\}$ with the binary operation $*$, which is given in Table 1 .

Table 1. Cayley table for the binary operation " $*$ ".

\begin{tabular}{llll}
\hline$*$ & $\mathbf{0}$ & $\mathbf{1}$ & $\boldsymbol{a}$ \\
\hline 0 & 0 & 0 & $a$ \\
1 & 1 & 0 & $a$ \\
$a$ & $a$ & $a$ & 0 \\
\hline
\end{tabular}

Then the neutrosophic quadruple BCI-algebra $\mathcal{N}(U)$ has 81 elements. If we take $I=\{0, a\}$ and $J=\{0, a\}$, then

$$
\mathcal{N}(I, J)=\left\{\tilde{0}, \tilde{\beta}_{1}, \tilde{\beta}_{2}, \tilde{\beta}_{3}, \tilde{\beta}_{4}, \tilde{\beta}_{5}, \tilde{\beta}_{6}, \tilde{\beta}_{7}, \tilde{\beta}_{8}, \tilde{\beta}_{9}, \tilde{\beta}_{10}, \tilde{\beta}_{11}, \tilde{\beta}_{12}, \tilde{\beta}_{13}, \tilde{\beta}_{14}, \tilde{\beta}_{15}\right\}
$$

and it is routine to check that $\mathcal{N}(I, J)$ is a neutrosophic quadruple BCI-positive implicative ideal of $\mathcal{N}(U)$ where

$$
\begin{gathered}
\tilde{0}=(0,0 T, 0 I, 0 F), \tilde{\beta}_{1}=(0,0 T, 0 I, a F), \tilde{\beta}_{2}=(0,0 T, a I, 0 F), \tilde{\beta}_{3}=(0,0 T, a I, a F), \\
\tilde{\beta}_{4}=(0, a T, 0 I, 0 F), \tilde{\beta}_{5}=(0, a T, 0 I, a F), \tilde{\beta}_{6}=(0, a T, a I, 0 F), \tilde{\beta}_{7}=(0, a T, a I, a F),
\end{gathered}
$$




$$
\begin{aligned}
\tilde{\beta}_{8} & =(a, 0 T, 0 I, 0 F), \tilde{\beta}_{9}=(a, 0 T, 0 I, a F), \tilde{\beta}_{10}=(a, 0 T, a I, 0 F), \tilde{\beta}_{11}=(a, 0 T, a I, a F), \\
\tilde{\beta}_{12} & =(a, a T, 0 I, 0 F), \tilde{\beta}_{13}=(a, a T, 0 I, a F), \tilde{\beta}_{14}=(a, a T, a I, 0 F), \tilde{\beta}_{15}=(a, a T, a I, a F) .
\end{aligned}
$$

Proposition 1. Given nonempty subsets I and $J$ of $U$, the neutrosophic quadruple BCI-positive implicative ideal $\mathcal{N}(I, J)$ of $\mathcal{N}(U)$ satisfies the following assertions.

$$
\begin{aligned}
& (\forall \tilde{p}, \tilde{q}, \tilde{r} \in \mathcal{N}(U))(((\tilde{p} \boxminus \tilde{r}) \square \tilde{r}) \square(\tilde{q} \square \tilde{r}) \in \mathcal{N}(I, J) \Rightarrow(\tilde{p} \boxminus \tilde{q}) \square \tilde{r} \in \mathcal{N}(I, J)), \\
& (\forall \tilde{p}, \tilde{q} \in \mathcal{N}(U))(((\tilde{p} \square \tilde{q}) \square \tilde{q}) \square(\tilde{0} \square \tilde{q}) \in \mathcal{N}(I, J) \Rightarrow \tilde{p} \square \tilde{q} \in \mathcal{N}(I, J)) .
\end{aligned}
$$

Proof. Let $\mathcal{N}(I, J)$ be a neutrosophic quadruple BCI-positive implicative ideal of $\mathcal{N}(U)$ for any nonempty subsets $I$ and $J$ of $U$. Assume that $((\tilde{p} \sqcup \tilde{r}) \square \tilde{r}) \square(\tilde{q} \square \tilde{r}) \in \mathcal{N}(I, J)$ for all $\tilde{p}, \tilde{q}, \tilde{r} \in \mathcal{N}(U)$. Since

$$
\begin{aligned}
& (((\tilde{p} \sqcup \tilde{q}) \square \tilde{r}) \square \tilde{r}) \square(\tilde{0} \square \tilde{r})=(((\tilde{p} \sqcup \tilde{r}) \square \tilde{r}) \square \tilde{q}) \square((\tilde{q} \square \tilde{q}) \square \tilde{r}) \\
& =(((\tilde{p} \sqcup \tilde{r}) \square \tilde{r}) \sqcup \tilde{q}) \square((\tilde{q} \square \tilde{r}) \square \tilde{q}) \\
& \leq((\tilde{p} \boxminus \tilde{r}) \square \tilde{r}) \square(\tilde{q} \square \tilde{r}),
\end{aligned}
$$

we have $(((\tilde{p} \sqcup \tilde{q}) \square \tilde{r}) \square \tilde{r}) \square(\tilde{0} \square \tilde{r}) \in \mathcal{N}(I, J)$. Since $\mathcal{N}(I, J)$ is a neutrosophic quadruple BCI-positive implicative ideal, it follows that $(\tilde{p} \sqcup \tilde{q}) \square \tilde{r} \in \mathcal{N}(I, J))$. Hence (11) is valid. If we take $\tilde{q}=\tilde{0}$ and $\tilde{r}=\tilde{q}$ in (11), then we get (12).

We consider relations between neutrosophic quadruple ideal and neutrosophic quadruple BCI-positive implicative ideal.

Theorem 1. For any nonempty subsets I and $J$ of $U$, if $\mathcal{N}(I, J)$ is a neutrosophic quadruple BCI-positive implicative ideal of $\mathcal{N}(U)$, then it is a neutrosophic quadruple ideal of $\mathcal{N}(U)$.

Proof. Assume that $\mathcal{N}(I, J)$ is a neutrosophic quadruple BCI-positive implicative ideal of $\mathcal{N}(U)$. Let $\tilde{p}=\left(p_{1}, p_{2} T, p_{3} I, p_{4} F\right)$ and $\tilde{q}=\left(q_{1}, q_{2} T, q_{3} I, q_{4} F\right)$ be elements of $\mathcal{N}(U)$ such that $\tilde{q} \in \mathcal{N}(I, J)$ and $\tilde{p} \square \tilde{q} \in \mathcal{N}(I, J)$. Then

$$
((\tilde{p} \sqcup \tilde{0}) \square \tilde{0}) \square(\tilde{q} \square \tilde{0})=\tilde{p} \boxminus \tilde{q} \in \mathcal{N}(I, J),
$$

which implies that $\tilde{p}=\tilde{p} \square \tilde{0} \in \mathcal{N}(I, J)$. Therefore $\mathcal{N}(I, J)$ is a neutrosophic quadruple ideal of $\mathcal{N}(U)$.

The converse of Theorem 1 is not true as seen in the following example.

Example 2. Consider a BCI-algebra $U=\{0,1, a\}$ with the binary operation $*$, which is given in Table 2 .

Table 2. Cayley table for the binary operation " $*$ ".

\begin{tabular}{llll}
\hline$*$ & $\mathbf{0}$ & $\mathbf{1}$ & $\boldsymbol{a}$ \\
\hline 0 & 0 & 0 & 0 \\
1 & 1 & 0 & 0 \\
$a$ & $a$ & 1 & 0 \\
\hline
\end{tabular}

Then the neutrosophic quadruple BCI-algebra $\mathcal{N}(U)$ has 81 elements. If we take $I=\{0\}$ and $J=\{0\}$, then $\mathcal{N}(I, J)=\{\tilde{0}\}$ is a neutrosophic quadruple ideal of $\mathcal{N}(U)$. But it is not a neutrosophic quadruple $B C I$-positive implicative ideal of $\mathcal{N}(U)$ since

$$
(((a, a T, a I, a F) \square(1,1 T, 1 I, 1 F)) \square(1,1 T, 1 I, 1 F)) \square(\tilde{0} \square(1,1 T, 1 I, 1 F))=\tilde{0} \in \mathcal{N}(I, J)
$$


and $(a, a T, a I, a F) \sqcup(1,1 T, 1 I, 1 F)=(1,1 T, 1 I, 1 F) \notin \mathcal{N}(I, J)$.

Given nonempty subsets $I$ and $J$ of $U$, we provide conditions for the set $\mathcal{N}(I, J)$ to be a neutrosophic quadruple BCI-positive implicative ideal.

Theorem 2. If I and J are BCI-positive implicative ideal of $U$, then $\mathcal{N}(I, J)$ is a neutrosophic quadruple $B C I$-positive implicative ideal of $\mathcal{N}(U)$.

Proof. Assume that $I$ and $J$ are BCI-positive implicative ideal of $U$. Obviously $\tilde{0} \in \mathcal{N}(I, J)$. Let $\tilde{p}=\left(p_{1}, p_{2} T, p_{3} I, p_{4} F\right), \tilde{q}=\left(q_{1}, q_{2} T, q_{3} I, q_{4} F\right)$ and $\tilde{r}=\left(r_{1}, r_{2} T, r_{3} I, r_{4} F\right)$ be elements of $\mathcal{N}(U)$ such that $\tilde{q} \in \mathcal{N}(I, J)$ and $((\tilde{p} \sqcup \tilde{r}) \square \tilde{r}) \square(\tilde{q} \square \tilde{r}) \in \mathcal{N}(I, J)$. Then $q_{i} \in I$ and $q_{j} \in J$ for $i=1,2$ and $j=3$, 4 . Also

$$
\begin{aligned}
& ((\tilde{p} \sqcup \tilde{r}) \sqcup \tilde{r}) \sqcup(\tilde{q} \sqcup \tilde{r})=\left(\left(\left(p_{1}, p_{2} T, p_{3} I, p_{4} F\right) \sqcup\left(r_{1}, r_{2} T, r_{3} I, r_{4} F\right)\right) \sqcup\left(r_{1}, r_{2} T, r_{3} I, r_{4} F\right)\right) \\
& \square\left(\left(q_{1}, q_{2} T, q_{3} I, q_{4} F\right) \sqcup\left(r_{1}, r_{2} T, r_{3} I, r_{4} F\right)\right) \\
& =\left(\left(p_{1} * r_{1},\left(p_{2} * r_{2}\right) T,\left(p_{3} * r_{3}\right) I,\left(p_{4} * r_{4}\right) F\right) \sqcup\left(r_{1}, r_{2} T, r_{3} I, r_{4} F\right)\right) \\
& \square\left(q_{1} * r_{1},\left(q_{2} * r_{2}\right) T,\left(q_{3} * r_{3}\right) I,\left(q_{4} * r_{4}\right) F\right) \\
& =\left(\left(\left(p_{1} * r_{1}\right) * r_{1},\left(\left(p_{2} * r_{2}\right) * r_{2}\right) T,\left(\left(p_{3} * r_{3}\right) * r_{3}\right) I,\left(\left(p_{4} * r_{4}\right) * r_{4}\right) F\right)\right) \\
& \square\left(q_{1} * r_{1},\left(q_{2} * r_{2}\right) T,\left(q_{3} * r_{3}\right) I,\left(q_{4} * r_{4}\right) F\right) \\
& =\left(\left(\left(p_{1} * r_{1}\right) * r_{1}\right) *\left(q_{1} * r_{1}\right),\left(\left(\left(p_{2} * r_{2}\right) * r_{2}\right) *\left(q_{2} * r_{2}\right)\right) T\right. \text {, } \\
& \left.\left(\left(\left(p_{3} * r_{3}\right) * r_{3}\right) *\left(q_{3} * r_{3}\right)\right) I,\left(\left(\left(p_{4} * r_{4}\right) * r_{4}\right) *\left(q_{4} * r_{4}\right)\right) F\right) \\
& \in \mathcal{N}(I, J),
\end{aligned}
$$

and so $\left(\left(p_{i} * r_{i}\right) * r_{i}\right) *\left(q_{i} * r_{i}\right) \in I$ and $\left(\left(p_{j} * r_{j}\right) * r_{j}\right) *\left(q_{j} * r_{j}\right) \in J$ for $i=1,2$ and $j=3,4$. it follows from (10) that $p_{i} * r_{i} \in I$ and $p_{j} * r_{j} \in J$ for $i=1,2$ and $j=3,4$. Hence

$$
\begin{aligned}
\tilde{p} \boxminus \tilde{r} & =\left(p_{1}, p_{2} T, p_{3} I, p_{4} F\right) \triangleright\left(r_{1}, r_{2} T, r_{3} I, r_{4} F\right) \\
& =\left(p_{1} * r_{1},\left(p_{2} * r_{2}\right) T,\left(p_{3} * r_{3}\right) I,\left(p_{4} * r_{4}\right) F\right) \in \mathcal{N}(I, J) .
\end{aligned}
$$

Therefore $\mathcal{N}(I, J)$ is a neutrosophic quadruple BCI-positive implicative ideal of $\mathcal{N}(U)$.

Corollary 1. If I is a BCI-positive implicative ideal of $U$, then $\mathcal{N}(I)$ is a neutrosophic quadruple BCI-positive implicative ideal of $\mathcal{N}(U)$.

Theorem 3. Let I and $J$ be ideals of $U$ which satisfies the following condition.

$$
(\forall p, q \in U)(((p * q) * q) *(0 * q) \in I \cap J \Rightarrow p * q \in I \cap J) .
$$

Then $\mathcal{N}(I, J)$ is a neutrosophic quadruple BCI-positive implicative ideal of $\mathcal{N}(U)$.

Proof. Obviously $\tilde{0} \in \mathcal{N}(I, J)$. Let $\tilde{p}=\left(p_{1}, p_{2} T, p_{3} I, p_{4} F\right), \tilde{q}=\left(q_{1}, q_{2} T, q_{3} I, q_{4} F\right)$ and $\tilde{r}=\left(r_{1}, r_{2} T, r_{3} I\right.$, $\left.r_{4} F\right)$ be elements of $\mathcal{N}(U)$ such that $\tilde{r} \in \mathcal{N}(I, J)$ and $((\tilde{p} \sqcup \tilde{q}) \sqcup \tilde{q}) \sqcup(\tilde{r} \square \tilde{q}) \in \mathcal{N}(I, J)$. Then $r_{1}, r_{2} \in I$, $r_{3}, r_{4} \in J$ and

$$
\begin{aligned}
&((\tilde{p} \triangleright \tilde{q}) \square \tilde{q}) \square(\tilde{r} \square \tilde{q})=\left(\left(\left(p_{1} * q_{1}\right) * q_{1}\right) *\left(r_{1} * q_{1}\right),\left(\left(\left(p_{2} * q_{2}\right) * q_{2}\right) *\left(r_{2} * q_{2}\right)\right) T,\right. \\
&\left.\left(\left(\left(p_{3} * q_{3}\right) * q_{3}\right) *\left(r_{3} * q_{3}\right)\right) I,\left(\left(\left(p_{4} * q_{4}\right) * q_{4}\right) *\left(r_{4} * q_{4}\right)\right) F\right) \\
& \in \mathcal{N}(I, J),
\end{aligned}
$$


that is,

$$
\left(\left(p_{i} * q_{i}\right) * q_{i}\right) *\left(r_{i} * q_{i}\right) \in I \text { and }\left(\left(p_{j} * q_{j}\right) * q_{j}\right) *\left(r_{j} * q_{j}\right) \in J
$$

for $i=1,2$ and $j=3,4$. Note that

$$
\begin{aligned}
& \left(\left(\left(p_{k} * q_{k}\right) * q_{k}\right) *\left(0 * q_{k}\right)\right) *\left(\left(\left(p_{k} * q_{k}\right) * q_{k}\right) *\left(r_{k} * q_{k}\right)\right) \\
& \leq\left(r_{k} * q_{k}\right) *\left(0 * q_{k}\right) \leq r_{k} * 0=r_{k}
\end{aligned}
$$

for $k=1,2,3,4$ by (I), (1) and (4). Since $I$ and $J$ are ideals of $U$, it follows that

$$
\begin{aligned}
& \left(\left(\left(p_{i} * q_{i}\right) * q_{i}\right) *\left(0 * q_{i}\right)\right) *\left(\left(\left(p_{i} * q_{i}\right) * q_{i}\right) *\left(r_{i} * q_{i}\right)\right) \in I, \\
& \left(\left(\left(p_{j} * q_{j}\right) * q_{j}\right) *\left(0 * q_{j}\right)\right) *\left(\left(\left(p_{j} * q_{j}\right) * q_{j}\right) *\left(r_{j} * q_{j}\right)\right) \in J
\end{aligned}
$$

for $i=1,2$ and $j=3,4$. Combining (14) and (15), we get

$$
\left(\left(p_{i} * q_{i}\right) * q_{i}\right) *\left(0 * q_{i}\right) \in I \text { and }\left(\left(p_{j} * q_{j}\right) * q_{j}\right) *\left(0 * q_{j}\right) \in J
$$

for $i=1,2$ and $j=3,4$. Using (13) implies that $p_{i} * q_{i} \in I$ and $p_{j} * q_{j} \in J$ for $i=1,2$ and $j=3,4$. Thus

$$
\begin{aligned}
\tilde{p} \boxminus \tilde{q} & =\left(p_{1}, p_{2} T, p_{3} I, p_{4} F\right) \sqcup\left(q_{1}, q_{2} T, q_{3} I, q_{4} F\right) \\
& =\left(p_{1} * q_{1},\left(p_{2} * q_{2}\right) T,\left(p_{3} * q_{3}\right) I,\left(p_{4} * q_{4}\right) F\right) \in \mathcal{N}(I, J) .
\end{aligned}
$$

Therefore $\mathcal{N}(I, J)$ is a neutrosophic quadruple BCI-positive implicative ideal of $\mathcal{N}(U)$.

Corollary 2. Let I be an ideal of $U$ which satisfies the following condition.

$$
(\forall p, q \in U)(((p * q) * q) *(0 * q) \in I \Rightarrow p * q \in I) .
$$

Then $\mathcal{N}(I)$ is a neutrosophic quadruple BCI-positive implicative ideal of $\mathcal{N}(U)$.

Theorem 4. Let I and J be ideals of $U$ which satisfies the following condition.

$$
(\forall p, q, r \in U)(((p * r) *(q * r)) * r \in I \cap J \Rightarrow(p * q) * r \in I \cap J) .
$$

Then $\mathcal{N}(I, J)$ is a neutrosophic quadruple BCI-positive implicative ideal of $\mathcal{N}(U)$.

Proof. Suppose that $((p * q) * q) *(0 * q) \in I \cap J$ for all $p, q \in U$. Then $((p * q) *(0 * q)) * q=$ $((p * q) * q) *(0 * q) \in I \cap J$, which implies from (17) and (1) that $p * q=(p * 0) * q \in I \cap J$. Therefore $\mathcal{N}(I, J)$ is a neutrosophic quadruple BCI-positive implicative ideal of $\mathcal{N}(U)$ by Theorem 3.

Corollary 3. Let I be an ideal of $U$ which satisfies the following condition.

$$
(\forall p, q, r \in U)(((p * r) *(q * r)) * r \in I \Rightarrow(p * q) * r \in I) .
$$

Then $\mathcal{N}(I)$ is a neutrosophic quadruple BCI-positive implicative ideal of $\mathcal{N}(U)$.

Theorem 5. Let I and J be subsets of $U$ such that

$$
\begin{aligned}
& 0 \in I \cap J, \\
& (\forall p, q, r \in U)((((p * q) * q) *(0 * q)) * r \in I \cap J, r \in I \cap J \Rightarrow p * q \in I \cap J) .
\end{aligned}
$$

Then $\mathcal{N}(I, J)$ is a neutrosophic quadruple BCI-positive implicative ideal of $\mathcal{N}(U)$. 
Proof. If we take $q=0$ in (20) and use (1) and (III), then

$$
(\forall p, r \in U)(p * r \in I \cap J, r \in I \cap J \Rightarrow p \in I \cap J) .
$$

Hence $I$ and $J$ are ideals of $U$. Assume that $((p * q) * q) *(0 * q) \in I \cap J$ for all $p, q \in U$. Then

$$
(((p * q) * q) *(0 * q)) * 0=((p * q) * q) *(0 * q) \in I \cap J
$$

It follows from (19) and (20) that $p * q \in I \cap J$. Consequently, $\mathcal{N}(I, J)$ is a neutrosophic quadruple BCI-positive implicative ideal of $\mathcal{N}(U)$ by Theorem 3.

Corollary 4. Let I be a subset of U such that

$$
\begin{aligned}
& 0 \in I, \\
& (\forall p, q, r \in U)((((p * q) * q) *(0 * q)) * r \in I, r \in I \Rightarrow p * q \in I) .
\end{aligned}
$$

Then $\mathcal{N}(I)$ is a neutrosophic quadruple BCI-positive implicative ideal of $\mathcal{N}(U)$.

Theorem 6. Let I, J, G and $H$ be ideals of $U$ such that $G \subseteq I$ and $H \subseteq J$. If $G$ and $H$ are BCI-positive implicative ideals of $U$, then $\mathcal{N}(I, J)$ is a neutrosophic quadruple BCI-positive implicative ideal of $\mathcal{N}(U)$.

Proof. Let $p, q, r \in U$ be such that $((p * q) * q) *(0 * q) \in I \cap J$. Then

$$
\begin{aligned}
& (((p *(((p * q) * q) *(0 * q))) * q) * q) *(0 * q) \\
& =(((p * q) * q) *(0 * q)) *(((p * q) * q) *(0 * q)) \\
& =0 \in G \cap H
\end{aligned}
$$

and so $(p * q) *(((p * q) * q) *(0 * q))=(p *(((p * q) * q) *(0 * q))) * q \in G \cap H \subseteq I \cap J$ since $G$ and $H$ are BCI-positive implicative ideals of $U$. Thus $p * q \in I \cap J$, and therefore $\mathcal{N}(I, J)$ is a neutrosophic quadruple BCI-positive implicative ideal of $\mathcal{N}(U)$ by Theorem 3.

Corollary 5. Let I and $G$ be ideals of $U$ such that $G \subseteq I$. If $G$ is a BCI-positive implicative ideal of $U$, then $\mathcal{N}(I)$ is a neutrosophic quadruple BCI-positive implicative ideal of $\mathcal{N}(U)$.

Theorem 7. Let $I, J, G$ and $H$ be ideals of $U$ such that $G \subseteq I, H \subseteq J$ and

$$
(\forall p, q \in U)(((p * q) * q) *(0 * q) \in G \cap H \Rightarrow p * q \in G \cap H) .
$$

Then $\mathcal{N}(I, J)$ is a neutrosophic quadruple BCI-positive implicative ideal of $\mathcal{N}(U)$.

Proof. Let $p, q, r \in U$ be such that $r \in G \cap H$ and $((p * q) * q) *(r * q) \in G \cap H$. Since

$$
(((p * q) * q) *(0 * q)) *(((p * q) * q) *(r * q)) \leq(r * q) *(0 * q) \leq r * 0=r \in G \cap H,
$$

we have $((p * q) * q) *(0 * q) \in G \cap H$. It follows from (23) that $p * q \in G \cap H$. Hence $G$ and $H$ are BCI-positive implicative ideals of $U$, and therefore $\mathcal{N}(I, J)$ is a neutrosophic quadruple BCI-positive implicative ideal of $\mathcal{N}(U)$ by Theorem 6.

Corollary 6. Let I and $G$ be ideals of $U$ such that $G \subseteq I$ and

$$
(\forall p, q \in U)(((p * q) * q) *(0 * q) \in G \Rightarrow p * q \in G)
$$

Then $\mathcal{N}(I)$ is a neutrosophic quadruple BCI-positive implicative ideal of $\mathcal{N}(U)$. 
Theorem 8. Let $I, J, G$ and $H$ be ideals of $U$ such that $G \subseteq I, H \subseteq J$ and

$$
(\forall p, q \in U)(((p * r) *(q * r)) * r \in G \cap H \Rightarrow(p * q) * r \in G \cap H) .
$$

Then $\mathcal{N}(I, J)$ is a neutrosophic quadruple BCI-positive implicative ideal of $\mathcal{N}(U)$.

Proof. Let $p, q \in U$ be such that $((p * q) * q) *(0 * q) \in G \cap H$. Then

$$
((p * q) *(0 * q)) * q=((p * q) * q) *(0 * q) \in G \cap H
$$

It follows from (25) and (1) that $p * q=(p * 0) * q \in G \cap H$. Hence $\mathcal{N}(I, J)$ is a neutrosophic quadruple BCI-positive implicative ideal of $\mathcal{N}(U)$ by Theorem 7 .

Proof. If we put $q=0$ and $r=q$ in (25), then we have the condition (23). Hence $\mathcal{N}(I, J)$ is a neutrosophic quadruple BCI-positive implicative ideal of $\mathcal{N}(U)$ by Theorem 7 .

Corollary 7. Let I and $G$ be ideals of $U$ such that $G \subseteq I$ and

$$
(\forall p, q \in U)(((p * r) *(q * r)) * r \in G \Rightarrow(p * q) * r \in G) .
$$

Then $\mathcal{N}(I)$ is a neutrosophic quadruple BCI-positive implicative ideal of $\mathcal{N}(U)$.

\section{Conclusions}

By considering an entry (i.e., a number, an idea, an object, etc.) which is represented by a known part $(a)$ and an unknown part $(b T, c I, d F)$ where $T, I, F$ have their usual neutrosophic logic meanings and $a, b, c, d$ are real or complex numbers, Smarandache have introduced the concept of neutrosophic quadruple numbers. Using the notion of neutrosophic quadruple numbers based on a set (instead of real or complex numbers), Jun et al. have constructed neutrosophic quadruple BCK/BCI-algebras and implicative neutrosophic quadruple BCK-algebras. In this manuscript, we have introduced the concept of a neutrosophic quadruple BCI-positive implicative ideal, and investigated several properties. We have discussed relations between neutrosophic quadruple ideal and neutrosophic quadruple BCI-positive implicative ideal. Given nonempty subsets $I$ and $J$ of a BCI-algebra $U$, we have provided conditions for the neutrosophic quadruple $(I, J)$-set to be a neutrosophic quadruple BCI-positive implicative ideal. In the forthcoming research and papers, we will continue these ideas and will define new notions in several algebraic structures.

Author Contributions: These authors contributed equally to this work. All authors have read and approved the final manuscript for submission.

Funding: The second author, Seok-Zun Song, was supported by Basic Science Research Program through the National Research Foundation of Korea (NRF) funded by the Ministry of Education (No. 2016R1D1A1B02006812).

Acknowledgments: We would like to thank anonymous reviewers for their very careful reading and valuable comments and suggestions.

Conflicts of Interest: The authors declare no conflict of interest.

\section{References}

1. Iséki, K. On BCI-algebras. Math. Semin. Notes 1980, 8, 125-130.

2. Iséki, K.; Tanaka, S. An introduction to the theory of BCK-algebras. Math. Jpn. 1978, 23, 1-26.

3. Borumand Saeid, A.; Jun, Y.B. Neutrosophic subalgebras of BCK/BCI-algebras based on neutrosophic points. Ann. Fuzzy Math. Inform. 2017, 14, 87-97. [CrossRef]

4. Jun, Y.B. Neutrosophic subalgebras of several types in BCK/BCI-algebras. Ann. Fuzzy Math. Inform. 2017, 14, 75-86. 
5. Jun, Y.B.; Kim, S.J.; Smarandache, F. Interval neutrosophic sets with applications in BCK/BCI-algebra. Axioms 2018, 7, 23. [CrossRef]

6. Jun, Y.B.; Smarandache, F.; Bordbar, H. Neutrosophic $\mathcal{N}$-structures applied to BCK/BCI-algebras. Information 2017, 8, 128. [CrossRef]

7. Jun, Y.B.; Smarandache, F.; Song, S.Z.; Khan, M. Neutrosophic positive implicative $\mathcal{N}$-ideals in BCK/BCI-algebras. Axioms 2018, 7, 3. [CrossRef]

8. Khan, M.; Anis, S.; Smarandache, F.; Jun, Y.B. Neutrosophic $\mathcal{N}$-structures and their applications in semigroups. Ann. Fuzzy Math. Inform. 2017, 14, 583-598.

9. Öztürk, M.A.; Jun, Y.B. Neutrosophic ideals in BCK/BCI-algebras based on neutrosophic points. J. Int. Math. Virtual Inst. 2018, 8, 1-17.

10. Song, S.Z.; Smarandache, F.; Jun, Y.B. Neutrosophic commutative $\mathcal{N}$-ideals in BCK-algebras. Information 2017, 8, 130. [CrossRef]

11. Smarandache, F. Neutrosophy, Neutrosophic Probability, Set, and Logic, ProQuest Information E Learning; American Research Press: Rehoboth, DE, USA, 1998; 105p. Available online: http://fs.gallup.unm.edu/ eBook-neutrosophics6.pdf (accessed on 27 March 2019).

12. Smarandache, F. A Unifying Field in Logics: Neutrosophic Logic. Neutrosophy, Neutrosophic Set, Neutrosophic Probability; American Reserch Press: Rehoboth, NM, USA, 1999.

13. Smarandache, F. Neutrosophic set-A generalization of the intuitionistic fuzzy set. Int. J. Pure Appl. Math. 2005, 24, 287-297.

14. Smarandache, F. Neutrosophic quadruple numbers, refined neutrosophic quadruple numbers, absorbance law, and the multiplication of neutrosophic quadruple numbers. Neutrosophic Sets Syst. 2015, 10, 96-98.

15. Jun, Y.B.; Song, S.Z.; Smarandache, F.; Bordbar, H. Neutrosophic quadruple BCK/BCI-algebras. Axioms 2018, 7, 41. [CrossRef]

16. Muhiuddin, G.; Al-Kenani, A.N.; Roh, E.H.; Jun, Y.B. Implicative neutrosophic quadruple BCK-algebras and ideals. Symmetry 2019, 11, 277. [CrossRef]

17. Huang, Y. BCI-Algebra; Science Press: Beijing, China, 2006.

18. Liu, Y.L.; Xu, Y.; Meng, J. BCI-implicative ideals of BCI-algebras. Inform. Sci. 2007, 177, 4987-4996. [CrossRef]

19. Liu, Y.L.; Zhang, X.H. Characterization of weakly positive implicative BCI-algebras. J. Hanzhong Teach. Coll. 1994, 1, 4-8.

20. Meng, J.; Jun, Y.B. BCK-Algebras; Kyungmoonsa Co.: Seoul, Korea, 1994.

21. Neutrosophy, Neutrosophic and Applications. Availiable online: http://fs.gallup.unm.edu/neutrosophy. htm (accessed on 27 March 2019)

(C) 2019 by the authors. Licensee MDPI, Basel, Switzerland. This article is an open access article distributed under the terms and conditions of the Creative Commons Attribution (CC BY) license (http:/ / creativecommons.org/licenses/by/4.0/). 